\title{
Holomorphic solutions of the susy grassmannian $\sigma$-model and gauge invariance
}

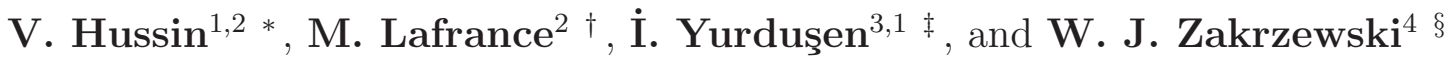 \\ ${ }^{1}$ Centre de Recherches Mathématiques, Université de Montréal, \\ CP 6128, Succ. Centre-Ville, Montréal, Quebec H3C 3J7, Canada \\ ${ }^{2}$ Département de Mathématiques et de Statistique, Université de Montréal, \\ CP 6128, Succ. Centre-Ville, Montréal, Quebec H3C 3J7, Canada \\ ${ }^{3}$ Department of Mathematics, Hacettepe University, \\ 06800 Beytepe, Ankara, Turkey \\ ${ }^{4}$ Department of Mathematical Sciences, University of Durham, \\ Durham DH1 3LE, United Kingdom
}

September 10, 2018

\begin{abstract}
For the first time we develop the gauge invariance of the supersymmetric grassmannian sigma model $G(M, N)$. It is richer then its purely bosonic submodel and we show how to use it in order to reduce some constant curvature holomorphic solutions of the model into simpler expressions.
\end{abstract}

Key words: supersymmetry (susy), grassmannian sigma models, gauge invariance PACS numbers: 12.60.Jv, 02.10.Ud, 02.10.Yn

\section{Introduction}

Although gauge invariance of the supersymmetric grassmannian sigma model (susy $\sigma$ model) $G(M, N)$ is well known [1, 2, 3, 4, to our knowledge, up to now no explicit form of it had been used in an effective way to analyse the solutions of the model. However, as the gauge invariance of the susy $G(M, N) \sigma$-model is richer than of its purely bosonic submodel, we can exploit this invariance to construct the solutions of the model in a simpler form. The aim of this manuscript is to demonstrate this fact explicitly. In order to make it self consistent we start by reminding the reader some properties of the susy $G(M, N) \sigma$-model [2]:

\footnotetext{
*E-mail address: hussin@dms.umontreal.ca

${ }^{\dagger}$ E-mail address: marie.lafrance@umontreal.ca

‡E-mail address: yurdusen@hacettepe.edu.tr

$\S$ E-mail address: w.j.zakrzewski@durham.ac.uk
} 
The susy $G(M, N) \sigma$-model is defined on a two-dimensional complex superspace $\left(x_{ \pm} ; \theta_{ \pm}\right)$, where $\left(x_{+}, x_{-}\right)$are local coordinates on $\mathbb{C}$ and $\left(\theta_{+}, \theta_{-}\right)$are complex odd Grassmann variables. The bosonic superfield has the following expansion

$$
\Phi\left(x_{ \pm}, \theta_{ \pm}\right)=\Phi_{0}\left(x_{ \pm}\right)+i \theta_{+} \Phi_{1}\left(x_{ \pm}\right)+i \theta_{-} \Phi_{2}\left(x_{ \pm}\right)-\theta_{+} \theta_{-} \Phi_{3}\left(x_{ \pm}\right),
$$

where $\Phi_{0}$ and $\Phi_{3}$ are $N \times M$ bosonic complex matrices and $\Phi_{1}$ and $\Phi_{2}$ are $N \times M$ fermionic complex matrices. As in a purely bosonic model [5], the superfield $\Phi$ satisfies

$$
\Phi^{\dagger} \Phi=I_{M} .
$$

The energy action functional of the model is given by

$$
S=2 \int_{S^{2}} d x_{+} d x_{-} d \theta_{+} d \theta_{-} \operatorname{Tr}\left(\left|\check{D}_{+} \Phi\right|^{2}-\left|\check{D}_{-} \Phi\right|^{2}\right),
$$

where the supercovariant derivatives $\check{D}_{ \pm}$are defined by

$$
\check{D}_{ \pm}=\check{\partial}_{ \pm}-\left(\Phi^{\dagger} \check{\partial}_{ \pm} \Phi\right)
$$

with usual superderivatives

$$
\check{\partial}_{ \pm}=-i \partial_{\theta_{ \pm}}+\theta_{ \pm} \partial_{ \pm}, \quad \partial_{ \pm} \equiv \partial_{x_{ \pm}} .
$$

Note that the susy operators satisfy $\check{\partial}_{ \pm}^{2}=-i \partial_{ \pm}$. Using the principle of least action, it is found that the superfield $\Phi$ satisfies the Euler-Lagrange equations of the model

$$
\check{D}_{+} \check{D}_{-} \Phi+\Phi\left|\check{D}_{-} \Phi\right|^{2}=0,
$$

together with the constraint (1.2). We know that if we want to obtain finite action solutions of the susy $G(M, N) \sigma$-model, we have to impose additionally the boundary conditions $\check{D}_{ \pm} \Phi \rightarrow 0,\left|x_{ \pm}\right| \rightarrow \infty$.

In the following section we use the well known MacFarlane parametrization (first used for finding solutions of the purely bosonic models [6], and later used also in the susy case [7]) and look at the solutions of the susy $G(M, N) \sigma$-model. In Section 3 , we show that these susy solutions can be effectively transformed into a simpler form by explicitly using the full gauge invariance of the model. In Section 4 , two examples are presented to emphasize the effectiveness of using the gauge transformation in the context of susy $G(M, N) \sigma$-model. Finally, we give some concluding remarks and discussions of our ongoing projects.

\section{MacFarlane parametrization and solutions of the susy $G(M, N) \sigma$-model}

Let us start with a holomorphic solution of the susy $G(M, N) \sigma$-model, written as

$$
W\left(x_{+}, \theta_{+}\right)=Z\left(x_{+}\right)+i \theta_{+} \eta\left(x_{+}\right) A\left(x_{+}\right),
$$

where we have introduced a fermionic function $\eta\left(x_{+}\right)$so that the matrices $Z \in \mathbb{C}^{N \times M}$ and $A \in \mathbb{C}^{N \times M}$ are both usual bosonic matrix functions of $x_{+}$. Moreover, the form of $Z$ is given by

$$
Z=\left(\begin{array}{c}
I_{M} \\
K
\end{array}\right), \quad K \in \mathbb{C}^{(N-M) \times M}
$$

The form (2.2) guarantees that $Z\left(x_{+}\right)$is written in the usual MacFarlane parametrization (i.e.; if we drop the fermionic part of (2.1) we end with the holomorphic solution of the purely bosonic model). At this stage $A\left(x_{+}\right)$is arbitrary and a natural question that can be asked 
is: Would it be possible to simplify the form of (2.1) by exploiting the gauge invariance of the susy model?

Let us first observe that the solution $W$ given by (2.1) satisfies $\check{\partial}_{-} W=0$, where $\check{\partial}_{-}$is defined in (1.5). by

This form of the solution is equivalent to a solution of the original susy model (1.6) given

$$
\Phi\left(x_{ \pm}, \theta_{ \pm}\right)=W\left(x_{+}, \theta_{+}\right) L\left(x_{ \pm}, \theta_{ \pm}\right),
$$

where $L\left(x_{ \pm}, \theta_{ \pm}\right)$is an $M \times M$ matrix. The function $\Phi$ has to satisfy the condition

$$
\Phi^{\dagger} \Phi=I_{M} \quad \Longleftrightarrow \quad W^{\dagger} W=\left(L L^{\dagger}\right)^{-1} .
$$

Proving that the expression for $\Phi\left(x_{ \pm}, \theta_{ \pm}\right)$given in (2.3), which comes from the holomorphic expression $W\left(x_{+}, \theta_{+}\right)$solves (1.6) is easy; here one repeats the steps used in the proof for the purely bosonic case. In order to see that $\check{\partial}_{-} W=0$ implies $\check{D}_{-} \Phi=0$, we consider

$$
\begin{aligned}
\check{D}_{-} \Phi & =\check{\partial}_{-} \Phi-\Phi\left(\Phi^{\dagger} \check{\partial}_{-} \Phi\right), \\
& =\left(1-\Phi \Phi^{\dagger}\right) \check{\partial}_{-} \Phi, \\
& =W\left(\check{\partial}_{-} L\right)-\Phi \Phi^{\dagger} W\left(\check{\partial}_{-} L\right), \\
& =W\left(\check{\partial}_{-} L\right)-W\left(L L^{\dagger}\right)\left(W^{\dagger} W\right)\left(\check{\partial}_{-} L\right)=0,
\end{aligned}
$$

due to (2.4).

In the purely bosonic case, this is the whole story; in the susy case $L$ is an $M \times M$ matrix superfield and so it makes sense to exploit this freedom to get a simpler expression for the additional matrix $A$. So let us look at the relations between $L$ and $A$ and, in particular, the implications of the right hand side of (2.4). Looking first at (2.1) we get

$$
W^{\dagger} W=Z^{\dagger} Z+i \theta_{+} \eta\left(Z^{\dagger} A\right)+i \theta_{-} \eta^{\dagger}\left(A^{\dagger} Z\right)-\theta_{+} \theta_{-} \eta^{\dagger} \eta\left(A^{\dagger} A\right) .
$$

To express the product $L L^{\dagger}$ we use the usual decomposition of a matrix superfield $L\left(x_{ \pm}, \theta_{ \pm}\right)$

$$
L=L_{0}+i \theta_{+} \eta L_{1}+i \theta_{-} \eta^{\dagger} L_{2}-\theta_{+} \theta_{-} \eta^{\dagger} \eta L_{3},
$$

together with the properties of Grassmann variables:

$$
\theta_{+}^{2}=\theta_{-}^{2}=0, \quad \theta_{+} \theta_{-}=-\theta_{-} \theta_{+}, \quad \theta_{+} \eta=-\eta \theta_{+}, \quad\left(\theta_{+} \eta\right)^{\dagger}=-\eta^{\dagger} \theta_{-} .
$$

This way we find the conditions which have to be imposed on the matrices $L_{0}, L_{1}, L_{2}$, and $L_{3}$ so that they satisfy (2.4):

$$
\begin{gathered}
L_{0} L_{0}^{\dagger}=M_{0}^{-1}, \\
L_{0} L_{2}^{\dagger}+L_{1} L_{0}^{\dagger}=-M_{0}^{-1} M_{1} M_{0}^{-1}, \\
L_{0} L_{1}^{\dagger}+L_{2} L_{0}^{\dagger}=-M_{0}^{-1} M_{2} M_{0}^{-1}, \\
L_{0} L_{3}^{\dagger}+L_{3} L_{0}^{\dagger}+L_{1} L_{1}^{\dagger}+L_{2} L_{2}^{\dagger}=-M_{0}^{-1}\left(M_{3}-M_{1} M_{0}^{-1} M_{2}-M_{2} M_{0}^{-1} M_{1}\right) M_{0}^{-1},
\end{gathered}
$$

where

$$
M_{0}=Z^{\dagger} Z, \quad M_{1}=Z^{\dagger} A, \quad M_{2}=A^{\dagger} Z, \quad M_{3}=A^{\dagger} A .
$$

Clearly $L_{0}$ is obtained from the purely bosonic solution $Z$. The two equations, involving $L_{1}$ and $L_{2}$ only are equivalent, since $M_{2}^{\dagger}=M_{1}$. As we are looking at the conditions that have to be imposed on the matrices $L_{i}$ we observe that, without any loss of generality, we can assume $L_{1}=0$ and $L_{3}=L_{3}^{\dagger}$ and we will satisfy all conditions on $L$. Indeed we find that

$$
L_{2}=-M_{0}^{-1} M_{1}^{\dagger} L_{0},
$$

and

$$
L_{0} L_{3}+L_{3} L_{0}=-M_{0}^{-1}\left(M_{3}-M_{1} M_{0}^{-1} M_{1}^{\dagger}\right) M_{0}^{-1} .
$$

In the subsequent development we will not need the explicit form of $L$. However, the choice of $L_{1}=0$ will play an important role in simplifying the final expressions. 


\section{Gauge invariance of the susy $G(M, N) \sigma$-model}

The gauge invariance of the susy $G(M, N) \sigma$-model is obtained by generalising its purely bosonic submodel to simplify the form of the solutions of the model.

If $\Phi$ is a solution of (1.6), then it can be shown that

$$
\tilde{\Phi}=V \Phi U,
$$

is also a solution where $V=V_{0} \in U(N)$ is a constant matrix (purely bosonic) and $U=$ $U\left(x_{ \pm}, \theta_{ \pm}\right) \in U(M)$. It is easy to see why $V_{0}$ has to be a constant matrix. Indeed we note that the supercovariant derivatives given in (1.4) and the superfield $\Phi$ transform in a similar fashion under the gauge transformation. Hence, starting with a general transformation matrix $V=V\left(x_{ \pm}, \theta_{ \pm}\right)$the imposition of the condition that $\Phi$ and $\check{D}_{ \pm} \Phi$ transform in the same way, we immediately obtain that $V$ is a constant matrix. This result is similar to the purely bosonic case $[5$. So let us consider the conditions on $U$.

Since the matrix $U$ depends on $x_{ \pm}$and also on $\theta_{ \pm}$, we can perform the usual decomposition

$$
U=U_{0}+i \theta_{+} \eta U_{1}+i \theta_{-} \eta^{\dagger} U_{2}-\theta_{+} \theta_{-} \eta^{\dagger} \eta U_{3},
$$

where now $U_{i}=U_{i}\left(x_{+}, x_{-}\right) \in \mathbb{C}^{M \times M}, i=0,1,2,3$ and the fermionic function $\eta\left(x_{+}\right)$is the same as before.

The condition of unitarity of $U$ may be written explicitly as

$$
U^{\dagger} U=U U^{\dagger}=I_{M} \Longleftrightarrow\left\{\begin{array}{c}
U_{0}^{\dagger}=U_{0}^{-1}, \quad U_{1}=-U_{0} U_{2}^{\dagger} U_{0}, \\
U_{3}+U_{0} U_{1}^{\dagger} U_{1}+U_{1} U_{1}^{\dagger} U_{0}+U_{0} U_{3}^{\dagger} U_{0}=0 .
\end{array}\right.
$$

Now, we address the question of how to exploit gauge invariance to reduce the solution $\Phi$ and thus $W$ to simpler expressions. Since we want to preserve the form of the purely bosonic solution $Z$, we consider the simplified solution in the form

$$
\Phi_{R}=W_{R} L_{R}=\left(Z+i \theta_{+} \eta A_{R}\right) L_{R},
$$

and want to find $V=V_{0}$ and $U$ such that

$$
\Phi_{R}=V_{0} \Phi U, \quad \Longleftrightarrow W_{R} L_{R}=V_{0} W L U .
$$

Writing $W_{R}$ and $W$ explicitly (3.5) becomes

$$
\left(Z+i \theta_{+} \eta A_{R}\right) L_{R}=V_{0}\left(Z+i \theta_{+} \eta A\right) L U .
$$

Considering first the purely bosonic case, we see that we can take $V_{0}=I_{N}$ and $U_{0}=I_{M}$. This implies that the purely bosonic part of $L_{R}$ is $L_{0 R}=L_{0}$. Note that now the unitarity of $U$ implies that

$$
U U^{\dagger}=U^{\dagger} U=I_{M} \Longleftrightarrow U_{1}=-U_{2}^{\dagger}, \quad U_{3}^{\dagger}+U_{3}+U_{1} U_{1}^{\dagger}+U_{1}^{\dagger} U_{1}=0 .
$$

Moreover, by choosing $U_{3}^{\dagger}=U_{3}$, we reduce the additional freedom in $U$ and get an explicit form of $U_{3}$ in terms of $U_{1}$ as

$$
U_{3}=-\frac{1}{2}\left(U_{1} U_{1}^{\dagger}+U_{1}^{\dagger} U_{1}\right) .
$$

It is important to observe here that the remaining gauge freedom is now reduced to the arbitrariness of choosing $U_{1}$.

Using the expressions of $V_{0}$ and $U$, we can now express the system (3.6) as a set of 3 matrix equations by identifying the coefficients of $\theta_{-}, \theta_{+}$and $\theta_{+} \theta_{-}$, respectively,

$$
\begin{gathered}
Z L_{2}+Z L_{0} U_{2}=Z L_{2 R} \\
Z L_{1}+Z L_{0} U_{1}+A L_{0}=Z L_{1 R}+A_{R} L_{0} \\
Z L_{3}+Z L_{0} U_{3}+Z L_{1} U_{2}+Z L_{2} U_{1}+A L_{2}+A L_{0} U_{2}=Z L_{3 R}+A_{R} L_{2 R} .
\end{gathered}
$$


Since $Z$ is in the MacFarlane parametrization (2.2), each of these equations can be split into two by taking into account the expressions for $A$ and $A_{R}$

$$
A=\left(\begin{array}{c}
\alpha \\
\beta
\end{array}\right), \quad A_{R}=\left(\begin{array}{c}
\alpha_{R} \\
\beta_{R}
\end{array}\right), \quad \begin{aligned}
& \alpha, \alpha_{R} \in \mathbb{C}^{M \times M}, \\
& \beta, \beta_{R} \in \mathbb{C}^{(N-M) \times M} .
\end{aligned}
$$

The final form of our equations 3.9 3.11) is then

$$
\begin{gathered}
L_{2 R}=L_{2}-L_{0} U_{1}^{\dagger}, \\
L_{1 R}=L_{1}+L_{0} U_{1}+\left(\alpha-\alpha_{R}\right) L_{0}, \\
\beta_{R}=\beta-K\left(\alpha-\alpha_{R}\right), \\
L_{3 R}=L_{3}+L_{0} U_{3}+L_{2} U_{1}-L_{1} U_{1}^{\dagger}-\alpha_{R} L_{2 R}-\alpha L_{0} U_{1}^{\dagger} .
\end{gathered}
$$

Since (3.15) does not involve $U_{1}$, the matrix function $\beta$ cannot be modified further by using the gauge freedom. The only equation involving $U_{1}$ and $\alpha$, in a simple way, is (3.14). Hence, assuming $L_{1}=L_{1 R}=0$ we get

$$
U_{1}=-L_{0}^{-1}\left(\alpha-\alpha_{R}\right) L_{0}
$$

This enables us to put $\alpha_{R}=0$ in the simplified form of $A_{R}$ which greatly simplifies the arbitrariness of $A$ and hence of the solution of the susy $G(M, N) \sigma$-model. Now, we have that

$$
U_{1}=-L_{0}^{-1} \alpha L_{0}
$$

together with

$$
\beta_{R}=\beta-K \alpha
$$

We see that by now, the gauge freedom has been completely used up and the explicit forms of $L_{2 R}$ and $L_{3 R}$ can be obtained from (3.13) and (3.16), respectively.

Hence, having started from a solution (2.1) in which $A$ is completely arbitrary we reach a simplified solution

$$
W_{R}=Z+i \theta_{+} \eta A_{R}
$$

with

$$
A_{R}=\left(\begin{array}{c}
0 \\
\beta-K \alpha
\end{array}\right)
$$

and this result holds in the gauge (3.18). So the simple form of the solution holds in this particular gauge.

\section{Constant curvature solutions and gauge invariance}

In our analysis of different sets of holomorphic solutions of the susy $G(M, N) \sigma$-models we have already found some of them generalising the purely bosonic ones. We consider now two examples in order to emphasize the importance and usefulness of the gauge invariance in the context of susy $G(M, N) \sigma$-model.

\subsection{Constant curvature solutions of $\mathbb{C} P^{N-1}$ model}

First we look at such solutions for the case of $M=1$ i.e.; of the $\mathbb{C} P^{N-1}$ model.

It has been shown that the susy holomorphic constant curvature solution of $\mathbb{C} P^{N-1}$ model can be written in the form 8

$$
\widetilde{W}\left(x_{+}, \theta_{+}\right)=u\left(x_{+}\right)+i \theta_{+} \eta\left(x_{+}\right) A\left(x_{+}\right),
$$


where $u\left(x_{+}\right)=\left(u_{n}\left(x_{+}\right)\right)$is the Veronese sequence with

$$
u_{n}\left(x_{+}\right)=\sqrt{\left(\begin{array}{c}
N-1 \\
n
\end{array}\right)} x_{+}^{n}, \quad n=0,1,2, \ldots, N-1,
$$

and $A\left(x_{+}\right)=\left(a_{n}\left(x_{+}\right)\right)$, with

$$
a_{n}\left(x_{+}\right)=-a_{0}\left(x_{+}\right)(n-1) u_{n}\left(x_{+}\right)+\frac{a_{1}\left(x_{+}\right)}{\sqrt{N-1}} \partial_{+} u_{n}\left(x_{+}\right), \quad n=0,1,2, \ldots, N-1 .
$$

We want to simplify this solution by applying to it a gauge transformation $U$, which is now a complex function of $\left(x_{ \pm}, \theta_{ \pm}\right)$. Indeed,

$$
U=1+i \theta_{+} \eta \tilde{u}\left(x_{ \pm}\right)+i \theta_{-} \eta^{\dagger} \tilde{u}^{*}\left(x_{ \pm}\right)-\theta_{+} \theta_{-} \eta^{\dagger} \eta\left|\tilde{u}\left(x_{ \pm}\right)\right|^{2} .
$$

From our preceding discussion, we see that the choice of the gauge $\tilde{u}$ is

$$
\tilde{u}=\alpha_{R}-\alpha,
$$

since $L_{0}$ is a scalar function. Due to the fact that we want $\alpha_{R}=0$, we see that

$$
\tilde{u}=-\alpha=-a_{0}\left(x_{+}\right) .
$$

Our ultimate goal is to find the expression for $A_{R}$ which appears in $\widetilde{W}_{R}$. Indeed, solving the equation

$$
\beta_{R}=\beta-K \alpha,
$$

in our special case, we get the final form of $A_{R}$ as

$$
a_{R n}=\frac{n}{\sqrt{N-1}} \frac{u_{n}\left(x_{+}\right)}{x_{+}}\left(a_{1}\left(x_{+}\right)-a_{0}\left(x_{+}\right) \sqrt{N-1} x_{+}\right), \quad n=0, \ldots, N-1 .
$$

This means that the unique susy holomorphic constant curvature solution of $\mathbb{C} P^{N-1}$ model is

$$
\widetilde{W}\left(x_{+}, \theta_{+}\right)=u\left(x_{+}\right)+i \theta_{+} \frac{\tilde{\eta}\left(x_{+}\right)}{\sqrt{N-1}} \partial_{+} u_{n}\left(x_{+}\right), \quad n=0, \ldots, N-1,
$$

where $\tilde{\eta}\left(x_{+}\right)=\left(a_{1}\left(x_{+}\right)-a_{0}\left(x_{+}\right) \sqrt{N-1} x_{+}\right)$is an arbitrary function of $x_{+}$, in agreement with our previous result [9]. That is the solution (4.9) given in our previous theorem 9 is unique up to gauge transformations.

\subsection{The simplest holomorphic constant curvature solution of $G(2,4)$ $\sigma$-model}

Considering the special solution $W_{1}$ of the form (2.1) with

$$
Z_{1}=\left(\begin{array}{c}
I_{2} \\
K_{1}
\end{array}\right), \quad K_{1}=\left(\begin{array}{cc}
x_{+} & 0 \\
0 & 0
\end{array}\right)
$$

it can be shown that $A$ takes the form [8]

$$
A_{1}=\left(\begin{array}{c}
\alpha \\
\beta
\end{array}\right)=\left(\begin{array}{cc}
\alpha_{11}\left(x_{+}\right) & \alpha_{12}\left(x_{+}\right) \\
\alpha_{21}\left(x_{+}\right) & \alpha_{22}\left(x_{+}\right) \\
\beta_{11}\left(x_{+}\right) & c_{0}+\left(c_{1}+\alpha_{12}\left(x_{+}\right)\right) x_{+} \\
b_{0}+b_{1} x_{+} & d_{0}
\end{array}\right),
$$

in order to have a constant curvature solution.

This solution $W_{1}$, can be reduced by using the gauge invariance (indeed using only $U_{1}$ given in (3.18) ) to the following form

$$
W_{1 R}=Z_{1}+i \theta_{+} \eta\left(x_{+}\right) A_{R}\left(x_{+}\right),
$$

with

$$
A_{R}=\left(\begin{array}{c}
0 \\
\beta_{R}-K_{1} \alpha
\end{array}\right), \quad \beta_{R}=\left(\begin{array}{cc}
\beta_{11}\left(x_{+}\right)-\alpha_{11}\left(x_{+}\right) x_{+} & c_{0}+c_{1} x_{+} \\
b_{0}+b_{1} x_{+} & d_{0}
\end{array}\right) .
$$




\section{Conclusions and final comments}

In this article we have shown that the gauge invariance of the susy $G(M, N) \sigma$-model can be used effectively to obtain simple forms of the holomorphic constant curvature solutions. Among other things this has allowed us to claim that:

- All solutions that can be obtained by direct calculations (e.g.; the examples of the constant curvature solutions for $\mathbb{C} P^{N-1} \sigma$-model and for $G(2,4) \sigma$-model) could be written in such a simplified form.

- In order to solve the general problem (say for $G(2,4)$ ), the computations can be started by assuming $A$ to be of the form (3.21).

We believe that exploiting the gauge invariance in such an effective way many properties of the solutions of the susy $G(M, N) \sigma$-model can be understood more easily. In particular, work is in progress on determining all constant curvature solutions of the susy $G(2,4) \sigma$ model in which all holomorphic bosonic solutions are given by:

$$
\begin{aligned}
& Z_{1}=\left[\begin{array}{cc}
1 & 0 \\
0 & 1 \\
x_{+} & 0 \\
0 & 0
\end{array}\right], Z_{2}=\left[\begin{array}{cc}
1 & 0 \\
0 & 1 \\
x_{+}^{2} \cos 2 t & \sqrt{2} x_{+} \cos t \\
\sqrt{2} x_{+} \sin t & 0
\end{array}\right], \\
& Z_{3}=\left[\begin{array}{cc}
1 & 0 \\
0 & 1 \\
\sqrt{3} x_{+}^{2} & \sqrt{8 / 3} x_{+} \\
0 & \sqrt{1 / 3} x_{+}
\end{array}\right], Z_{4}=\left[\begin{array}{cc}
1 & 0 \\
0 & 1 \\
2 x_{+}^{3} & \sqrt{3} x_{+}^{2} \\
\sqrt{3} x^{2} & 2 x_{+}
\end{array}\right],
\end{aligned}
$$

where $t$ is a real parameter. Up to a $U(4)$ gauge transformation, these are the only bosonic solutions with constant curvature [10.

Finally, the next step would be to analyse the constant curvature solutions of the susy $G(2,5)$ and in general the susy $G(2, N) \sigma$-models where the purely bosonic solutions are given in [11] and [12, respectively.

\section{Acknowledgments}

The work of IY was partly supported by Hacettepe University Scientific Research Coordination Unit. Project Number: FBI-2017-14035. He also thanks the Centre de Recherches Mathématiques, Université de Montréal for the kind hospitality while on sabbatical leave during which this work was done. $\mathrm{VH}$ acknowledges the support of research grants from NSERC of Canada.

\section{References}

[1] Witten E 1977 Supersymmetric form of the nonlinear $\sigma$ model in two dimensions Phys. Rev. D 16 2991-4

[2] Din A M, Lukierski J and Zakrzewski W J 1982 General classical-solutions of a supersymmetric non-linear coupled boson-fermion model in two dimensions Nucl. Phys. B 194 157-71

[3] Fujii K, Koikawa T and Sasaki R 1984 Classical Solutions for the Supersymmetric Grassmannian Sigma Models in Two Dimensions I Prog. Theor. Phys. 71 388-94

[4] Fujii K and Sasaki R 1984 Classical Solutions for the Supersymmetric Grassmannian Sigma Models in Two Dimensions II Prog. Theor. Phys. 71 831-9 
[5] Zakrzewski W J 1989 Low Dimensional Sigma Models (Bristol: Adam Hilger)

[6] MacFarlane A J 1978 Generalizations of $\sigma$-models and $\mathbb{C} P^{N}$ models and instantons Phys. Lett. B 82 239-41

[7] Din A M and Zakrzewski W J 1984 Further properties of classical solutions in grassmannian $\sigma$ models Nucl. Phys. B 237 461-76

[8] Lafrance M 2018 Solutions à courbure constante de modèles sigma supersymétriques (Master's thesis) Université de Montréal

[9] Delisle L, Hussin V, Yurduşen İ and Zakrzewski W J 2015 Constant curvature surfaces of the supersymmetric $\mathbb{C} P^{N-1}$ sigma model J. Math. Phys 56 023506-1-18

[10] Li Z Q and Yu Z H 1999 Constant curved minimal 2-spheres in G(2,4) Manuscripta Math. 100 305-16

[11] Jiao X X and Peng J G 2004 Classification of holomorphic spheres of constant curvature in complex Grassmann manifold G(2,5) Diff. Geo. Its Appl. 20 267-77

[12] Peng C K and Xu X W 2015 Classification of minimal homogeneous two-spheres in the complex Grassmann manifold G(2,n) J. Math. Pures Appl. 103 374-99 